\title{
]jfis
}

\section{Adaptive Image Segmentation Based on Histogram Transition Zone Analysis}

\author{
Rafael Guillermo González Acuña ${ }^{1}$, Domingo Mery ${ }^{2}$, and Reinhard Klette ${ }^{3}$ \\ ${ }^{1}$ Universidad Nacional Abierta y a Distancia de México, Monterrey, México \\ ${ }^{2}$ Department of Computer Science, Pontificia Universidad Católica de Chile, Santiago, Chile \\ ${ }^{3}$ Auckland University of Technology, Auckland, New Zealand
}

\begin{abstract}
While segmenting "complex" images (with multiple objects, many details, etc.) we experienced a need to explore new ways for time-efficient and meaningful image segmentation. In this paper we propose a new technique for image segmentation which has only one variable for controlling the expected number of segments. The algorithm focuses on the treatment of pixels in transition zones between various label distributions. Results of the proposed algorithm (e.g. on the Berkeley image segmentation dataset) are comparable to those of GMM or HMM-EM segmentation, but are achieved with significantly reduced computation time.
\end{abstract}

Keywords: image segmentation, image analysis, comparative evaluation

\section{Introduction}

Image segmentation is the basis of efficient image analysis and data extraction. Image segmentation algorithms partition the input images into pairwise disjoint regions. Segmentation results (i.e. the segments) can be used for object proposals [1, 2] which may define candidates for a classification module. Image segmentation methods follow different strategies such as region-based [3], active contours [4], clustering [5], histogram-based [6], graph-based [7], or deep learning [8]. Histogram-based methods do not require any prior information about the input images, and date back to the beginning of image analysis [9]. This paper proposes a histogram-based algorithm (called adaISA) for adaptive image segmentation.

Given an image, the algorithm uses only one parameter for the segmentation; adaISA

Received: Dec. 14, 2016

Revised : Dec. 19, 2016

Accepted: Dec. 22, 2016

Correspondence to: Rafael Guillermo González Acuña

(rafael123.90@hotmail.com)

(CThe Korean Institute of Intelligent Systems

(c) This is an Open Access article distributed under the terms of the Creative Commons Attribution Non-Commercial License (http://creativecommons.org/licenses/ by-nc/3.0// which permits unrestricted noncommercial use, distribution, and reproduction in any medium, provided the original work is properly cited. decides about this number by only analysing the histogram of image features. Our algorithm is applicable to both gray-level or colour images.

In general, considered features for image segmentation can be vectors combining $(x, y)$ pixel coordinates, pixel values, or low-level descriptors such as local means, variances, or estimated gradient values. For example, SLIC [10] combines coordinates $(x, y)$ and $\mathrm{L}^{\star} \mathrm{a}^{\star} \mathrm{b}^{\star}$ pixel values into 5-dimensional feature vectors, and mean-shift methods, e.g. discussed in [2], consider feature vectors defined by intensity values plus local means and variances. Feature vectors with $n \geq 1$ components define $n$-channel images; value distributions are commonly represented by $n$-dimensional feature histograms.

Our adaISA evolves from the common basic idea [9] that local minima in a feature histogram indicate border pixels between segments. Local minima in the histogram are basically at places where feature distributions intersect; adaISA pays special attention to the transition zones of feature distributions. 
We tested our approach for various definitions of feature spaces, and decided finally to break down the analysis of multichannel (feature) images into a subsequent analysis of singlechannel images; we combine then the results achieved for the single channels. This leads to results comparable to state-ofthe-art algorithms. We only have to deal with 1D histograms. adaISA does not consider feature vectors; instead we compute label images for single channels, and unify those into a single label image.

The paper is structured as follows. Section 2 specifies a basic single-channel image segmentation algorithm (ISA) for 1D histograms. This will then be refined in Section 3 into a Euclidean or Gaussian adaISA for a single channel; both versions are finally combined in Section 4 into Euclidean or Gaussian adaISA for multi-channel images. Section 5 provides qualitative and quantitative performance evaluation. Section 6 concludes.

\section{A Very Basic 1-Channel ISA}

Before discussing the proposed adaISA for multi-channel images, we start with a variant of a very basic adaptive ISA, similar to those histogram-based segmentation algorithms known from the early days of image processing [9].

The core idea of this basic 1-channel ISA can be easily summarised as follows: For every local minima $i$ in any of the 1D histograms $H$ of a multi-channel image, define a threshold $\tau_{i}$ at that position, then finish the scan of the respective histogram $H$ by putting all the thresholds into a threshold vector $v_{\text {th }}=\left[0, \tau_{1}, \tau_{2}, \ldots, \tau_{N}, 255\right]^{\top}$, where $N$ is the number of generated thresholds; for finding those we use Algorithm 1. Then merge the segment channels in a final segmented image; see Section 4

The chosen basic ISA does not perform local derivations of the 1D histogram for trying to find zeros at extrema (and for checking whether there is actually a local maxima or a local minima). Instead, the used basic ISA simply scans the whole histogram and finds local minima initiated by available global information.

First, this basic ISA applies a (small) mean filter to the histogram $H$ which is the convolution $H_{f}=H * K$ between $H$ and the kernel $K=[0.50 .5]$. Then it considers the filtered histogram $H_{f}$.

Second, this basic ISA scans the histogram with a scan line defined by a series of adjacent elements and checks whether there is a local minimum value in the considered interval. The

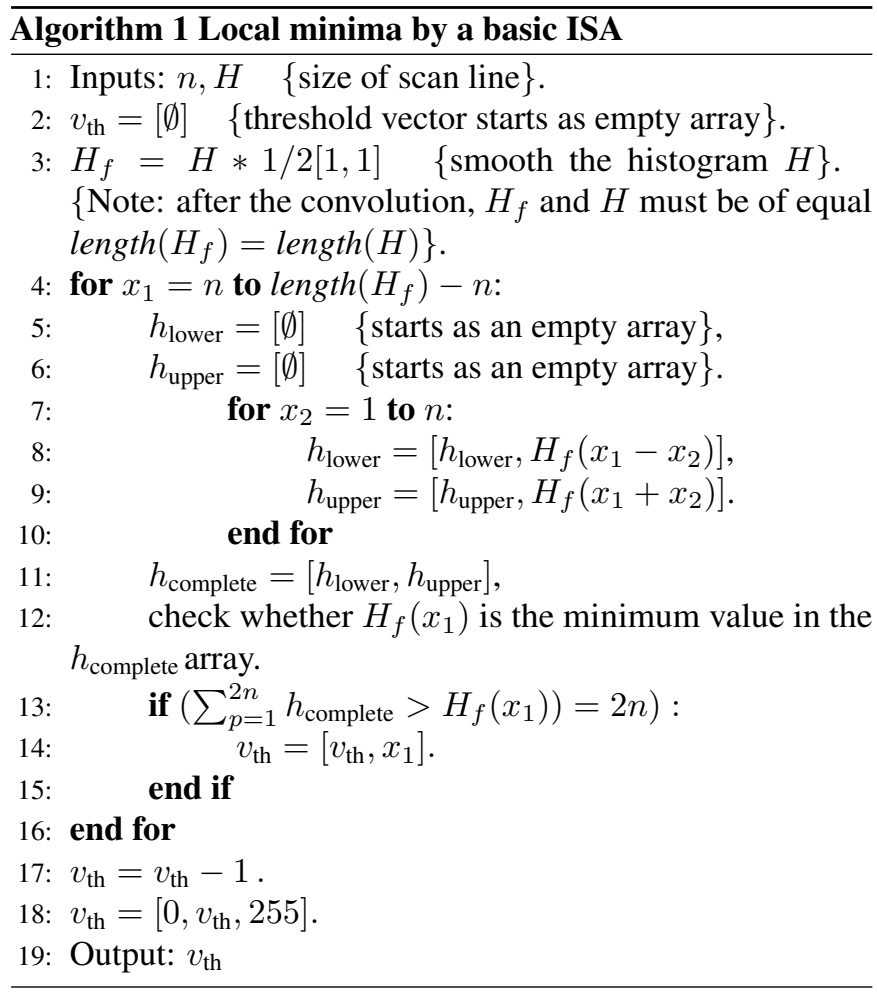

number of detected local minima is at this stage proportional to the size $2 \times n$ of the considered scan line, where $n$ is the only parameter of this basic ISA and is the half of the size of the scan line.

The basic ISA starts with two empty arrays $h_{\text {lower }}$ and $h_{\text {upper }}$ which are filled with the values corresponding to lower and upper values in the scan line. Algorithm 1 is the pseudocode of the used basic ISA. Note that the pseudo code has a similar notation as known from MATLAB.

At the end of Algorithm 1, $v_{\text {th }}$ is decremented by one since the histogram $H$ is a vector with 256 elements, where the index goes from 1 to 256, but the threshold must have a value between 0 and 255. This basic ISA has $n>0$ as its only parameter.

\section{Adaptive ISA with Transition Zones}

Transition zones of value distributions have been considered in various histogram-based ISAs, such as Otsu binarization [6] or the Gaussian mixture model (GMM) [12]. These methods aim at optimising a threshold under particular assumptions. The main problem of such algorithms is that a calculated threshold is not flexibly applied: A pixel with a given value can only belong to a segment defined by the pixel's value position relatively to the given threshold. We turn Algorithm 1 now into an adaptive ISA by providing a flexible decision for pixels in the transition 


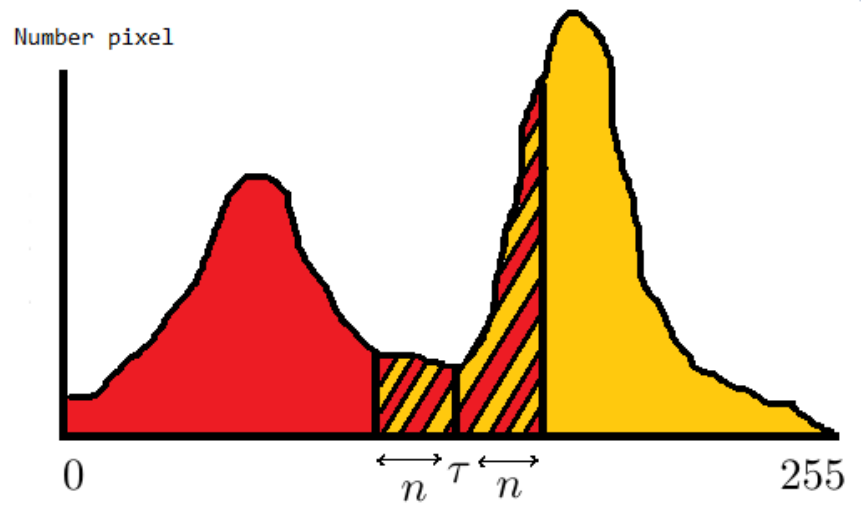

Figure 1. A histogram with a transition zone near $\tau$.

zones.

\subsection{Strategy for Transition Zones}

Transition zones are the areas of the histogram that enclose a threshold; see Fig. 1. In these zones, pixel values are not defining a unique assignment to one of the two adjacent peaks in value distributions. The size of the transition zone is defined by $n$, where the transition zone extends from the threshold position $\tau$ of the histogram and goes $n$ elements to the left of $\tau$, and $n$ elements to the right of $\tau$. The red zone and the orange zone are divided by threshold $\tau$. In the shown case we have a transition zone of size $2 \times n$. The assumed width of transition zones defines parameter $n$ in the basic ISA (i.e. Algorithm 1).

We want to identify pixels with values in these transition zones. For those pixels we decide to which of the two adjacent peaks they should actually be assigned to (and not only by simply applying the threshold at the given valley).

Pixels that contribute to the transition zone will be assigned individually to either the red segment or the orange one shown in Fig. 1. depending on "which segment is closer". We propose two methods for measuring "close", either by computing the Euclidean distance or by computing the probability using Gaussian distributions for values in the considered image channel.

\subsection{Use of Euclidean Distance}

We consider a 3D feature space, defined by pixel position $(x, y)$ and the selected single channel value for $I(x, y)$, for the given image $I$. First we compute the centroid (in this $3 \mathrm{D}$ feature space) of all the pixels which contribute to one of the two histogram segments that are to the left or right of the threshold. In the example of Fig. 1, we compute one centroid for the red segment, and one for the orange segment. Then we compute for each pixel $(x, y, I(x, y))$ with an assigned value in the transition zone the Euclidean distance

$$
\sqrt{\left[I(x, y)-\mu_{1}\right]^{2}+\left[x-\mu_{2}\right]^{2}+\left[y-\mu_{3}\right]^{2}},
$$

in the relevant 3D feature space to those two centroids, and assign it to segments defined by the closer centroid.

Assume that we have $N_{\text {segments }}$ clusters (or peaks) defined by the considered channel (i.e. the given 1D histogram $H$ ). Then, we need to consider $N_{\text {segments }}$ centroids defined by $\mu_{\alpha 1}$, the mean channel value of cluster $\alpha, \mu_{\alpha 2}$, the mean $x$-value of pixels that belong to cluster $\alpha$, and $\mu_{\alpha 2}$, the mean $y$-value of pixels that belong to cluster $\alpha$, with $\alpha \in\left\{1,2, \ldots, N_{\text {segments }}\right\}$. $N_{\text {segments }}$ is the number of thresholds obtained by Algorithm 1 plus one.

\subsection{Use of Gaussian Distributions}

In this case we model segments by Gaussian distributions that depend on the 1D channel values of the pixels and their positions $(x, y)$. In the example of Fig. 1. we sketch two Gaussian distributions, a red one and an orange one.

For every pixel inside of the transition zone we compute the probability to be part of one of the two "adjacent" Gaussian distributions. The pixel is then assigned to the segment with the Gaussian distribution were it has a higher probability to belong to. The Gaussian distribution is here of the form

$$
\frac{1}{(2 \pi)^{n / 2}\left|\Sigma_{\alpha}\right|^{1 / 2}} e^{-\frac{1}{2}\left[\mathbf{x}-\mu_{\alpha}\right]\left|\Sigma_{\alpha}\right|^{-1}\left[\mathbf{x}-\mu_{\alpha}\right]^{T}},
$$

where $\mathrm{x}$ is the vector that contains the $3 \mathrm{D}$ information of the pixel $(I(x, y), x, y)$ (i.e. it's channel value and coordinates $x$ and $y), \alpha$ is the label of the segment defined by $\alpha=$ $\left\{1,2, \ldots, N_{\text {segments }}\right\}$, where $N_{\text {segments }}$ is the number of segments, $\Sigma$ is the covariance matrix, and $\mu$ the mean vector.

\section{4. adaISA for Multi-Channel Images}

We specify the proposed algorithm adaISA now for multichannel (or multi-feature) images. We discuss a 3D image-value example, which is easy to generalise for any multi-channel situation. For images in $L a b$ space, we apply the single-channel method individually, for each colour channel $L^{\star}, a^{\star}$, and $b^{\star}$, which are the input images of Algorithm 1. We obtain label images $\Psi_{L}, \Psi_{a}$, and $\Psi_{b}$, which are the output images of Algorithm 1.

We unify those by applying Algorithm 2 . First, we merge the 


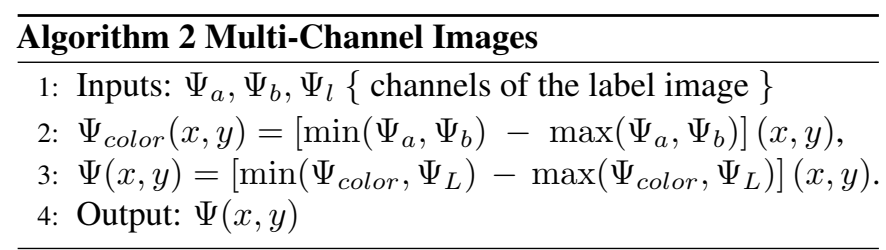

label images of both color channels $\Psi_{a}$ and $\Psi_{b}$ into one label image $\Psi_{\text {color }}$ representing information of the color channels. Then we merge $\Psi_{L}$ with $\Psi_{\text {color }}$ to generate the label image $\Psi$. This basic algorithm avoids any loops or iterations, and any complex operations. The performance of adaISA is very fast and allows us to obtain consistent results.

This algorithm is a modification of the blending Phoenix algorithm [11].

\section{Results of the Proposed Adaptive ISA}

We present results of the two versions of adaISA by using for transition zones either the Euclidean distance or Gaussian distributions. Input images are in $L a b$ space.

We compare both versions of adaISA with two common image segmentation algorithms based on the Gaussian mixture model (GMM) [12], or the hidden Markov model with expectation maximisation (HMM-EM) [13].

\subsection{Rand Index Criterion}

We evaluate similarity of results by using the Rand index criterion [14]. This criterion can be used for measuring the similarity between two label images $\Psi_{1}$ and $\Psi_{2}$ of identical size; normally we take $\Psi_{2}$ as the ground-truth image. Algorithm 3 follows Equ. (3) in the algorithm; it shows the steps for calculating this measure $\mathcal{R}\left(\Psi_{1}, \Psi_{2}\right)$, expressing the similarity between $\Psi_{1}$ and $\Psi_{2}$ in percent.

\subsection{Size of Transition Zones and Resolution of Segmenta- tion}

We test adaISA using different values of parameter $n$. Figure 4 shows input image El Castillo Stitch $2008^{4}$ and results of the Gaussian adaISA for $n=17$ and $n=9$. Figure 3 shows the input image Michoacan ${ }^{5}$ and results of the Euclidean adaISA for $n=3$ and $n=10$. Figure 3 shows the input image Foutain, also from the CCV data set, and

\footnotetext{
${ }^{4}$ Image in public domain, see https://commons. wikimedia.org

${ }^{5}$ From the CCV data set, publically available at ccv.wordpress.fos.auckland. ac.nz/data/single-images/
}
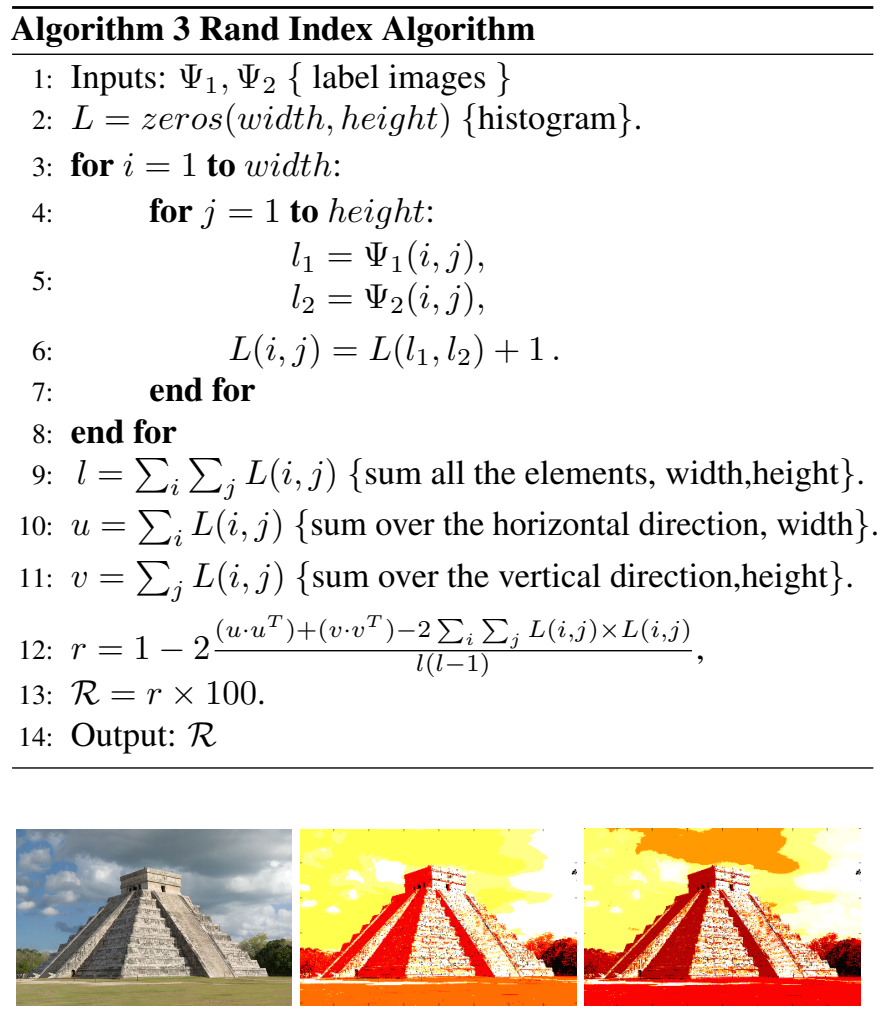

Figure 2. Left: Original image El Castillo Stitch 2008. Middle: Gaussian adaISA for $n=17$. Right: Gaussian adaISA for $n=9$
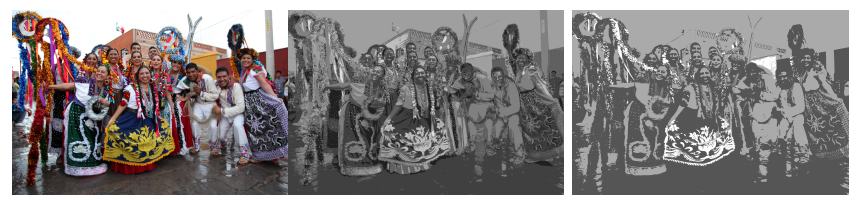

Figure 3. Left: Original image Michoacan. Middle: Euclidean adaISA for $n=3$. Right: Euclidean adaISA for $n=10$
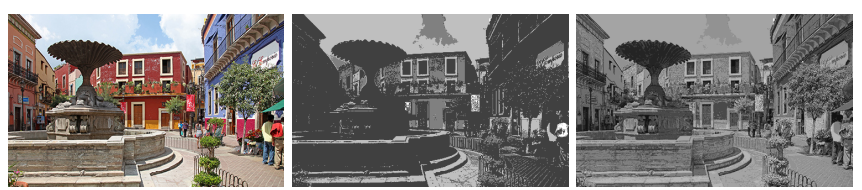

Figure 4. Left: Original image Fountain. Middle: Gaussian adaISA for $n=7$. Right: Gaussian adaISA for $n=3$

results of Gaussian adaISA with $n=7$ or $n=3$. All three input images are here shown as examples for "complex" scenes, showing diverse objects in structured environments,

Smaller values for $n>0$ produce in general a larger number of segments. Figure 2 is still too simple in structure for a good illustration of this effect.

In Fig. 3. $n=3$ allows us to subsegment the faces and the 
drawings on the dresses, and even the flag at the top of the building in the background, and $n=10$ produces just three segment categories; for example, floor and legs of the people are in the same segment category.

Figure 4 shows that information on the left (front of buildings) is lost for Gaussian adaISA with $n=7$, but $n=3$ allows us to assign segments to individual windows in this region. Parameter $n$, the only input parameter of our code, controls the resolution of the segmented image.

\subsection{Results and Comparison with Other Approaches}

In this section we apply adaISA with Gaussian distributions to the images of the Berkeley data set. In comparison, we also run methods based on GMM and HMM-EM on these images.

Table 1 lists Rand indices for estimating the similarity between results and the ground truth. For adaISA, the first column compares the Gaussian adaISA with ground truth adaISA, while the second column and the third column compares the GMM and HMM-EM with the ground truth, respectively.

Rand indices on the Berkeley data set demonstrate similarity between results obtained by Gaussian adaISA. Because adaISA has only a significant impact on pixels in transition zones, it is also no surprise that results for Gaussian adaISA are very similar to those obtained by HMM-EM segmentation.

From Table 1 we compute the mean, the standard deviation, the maximum value, the minimum value, and the median of the results for every code. Those values are presented in Table 2 .

Figure 5 presents a gallery of resulting images when Gaussian adaISA is applied to images of the Berkeley data set. The first column shows original images, the second shows segmentborder images, and the last column shows maps of labels of segment categories.

Figure 6 presents a gallery of resulting label maps for Gaussian adaISA, GMM segmentation, or HMM-EM segmentation for some of the images of the Berkeley data set. The first column shows (in gray-levels) label maps for the Gaussian adaISA, the second column (in blue colors) GMM label maps for, and the third column (in red colors) HMM-EM label maps. Euclidean adaISA is not shown in this gallery in Fig. 6 due to available space, but results of Gaussian or Euclidean ada ISA are similar.

Figure 7 presents a gallery of resulting images when Gaussian adaISA is applied to images of the Berkeley data set. The first column shows original images, the second shows ground truth images, and the last column shows maps of labels of segment categories.
Table 1. Rand indices for some of the images of the Berkeley data set. See text for explanation.

\begin{tabular}{|c|c|c|c|}
\hline Image name & ISA $\%$ & GMM\% & HMM-EM\% \\
\hline \# (167062) & 91 & 83 & 92 \\
\hline$\#(42049)$ & 83 & 78 & 88 \\
\hline$\#(62096)$ & 90 & 83 & 89 \\
\hline \# (101085) & 82 & 84 & 82 \\
\hline \# (241004) & 93 & 87 & 90 \\
\hline \# (37073) & 89 & 82 & 84 \\
\hline \# (296059) & 86 & 72 & 89 \\
\hline \# (145086) & 89 & 85 & 91 \\
\hline \# (119082) & 79 & 64 & 78 \\
\hline \# (208001) & 81 & 76 & 85 \\
\hline \# (189080) & 76 & 68 & 83 \\
\hline \# (160068) & 84 & 77 & 89 \\
\hline \# (147091) & 92 & 91 & 90 \\
\hline \#(3096) & 84 & 76 & 82 \\
\hline \# (302008) & 71 & 73 & 72 \\
\hline$\#(86016)$ & 93 & 83 & 80 \\
\hline$\#(24077)$ & 70 & 78 & 74 \\
\hline \# (198023) & 91 & 72 & 80 \\
\hline \# (181079) & 86 & 78 & 84 \\
\hline \# (198054) & 83 & 71 & 84 \\
\hline \# (135069) & 90 & 80 & 87 \\
\hline \# (388016) & 74 & 75 & 83 \\
\hline \# (182053) & 80 & 81 & 81 \\
\hline$\#(2100)$ & 70 & 73 & 70 \\
\hline$\#(21077)$ & 90 & 86 & 87 \\
\hline \# (376043) & 88 & 68 & 83 \\
\hline \# (106024) & 72 & 67 & 77 \\
\hline \# (302008) & 71 & 70 & 79 \\
\hline \# (197017) & 82 & 80 & 83 \\
\hline \# (299086) & 80 & 81 & 80 \\
\hline \# (241048) & 91 & 82 & 87 \\
\hline$\#(65033)$ & 85 & 80 & 90 \\
\hline \#(351093) & 82 & 80 & 84 \\
\hline \#(361010) & 83 & 81 & 86 \\
\hline$\#(8068)$ & 81 & 83 & 90 \\
\hline$\#(3063)$ & 94 & 80 & 90 \\
\hline \# (181021) & 86 & 81 & 84 \\
\hline
\end{tabular}



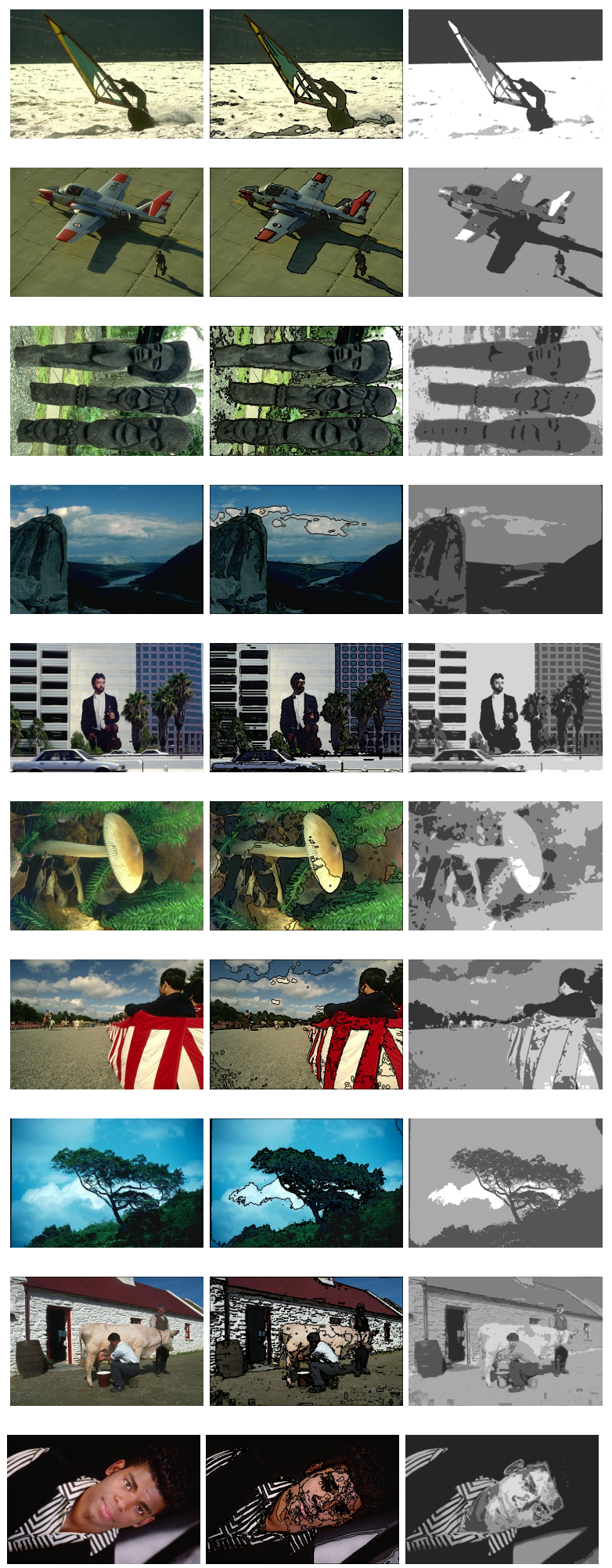

Figure 5. Resulting gallery of using Gaussian adaISA for some of the images of the Berkeley data set.
Table 2. Statistics of results for the images of the Berkeley data set of Table 1

\begin{tabular}{cccc}
\hline Variable & ISA\% & GMM\% & HMM\% \\
\hline mean & 82.4 & 78.08 & 83.97 \\
std & 7.15 & 6.13 & 5.36 \\
min & 70 & 64 & 70 \\
max & 94 & 91 & 92 \\
median & 84 & 80 & 84 \\
average run-time & $0.89 \mathrm{sec}$ & $9.78 \mathrm{sec}$ & $88.7 \mathrm{sec}$ \\
\hline
\end{tabular}
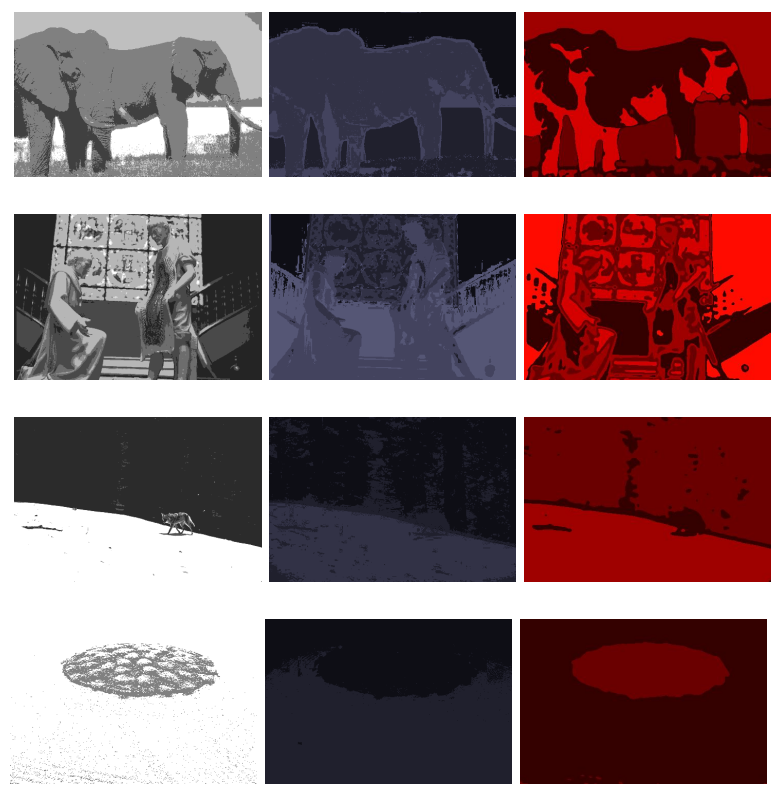

Figure 6. Gallery Left column: Gaussian adaISA, Center column: GMM Right column: HMM-EM.

\subsection{Segmentation of Aerial Images}

Our interest in segmentation is motivated by aerial image analysis, and we show finally a few examples from this area of application.

Figure 8 shows the bay of Cancun; the image Landsat_Cancun_ Mexico is available in the Wikimedia Commons data base. We illustrate in the figure that adaISA can segment independently the main regions of the bay such as the deeper ocean, the coast, the jungle, weed, the infrastructure, and also some boats on the ocean. The original image is on the left in the figure, the segment-border image is in the middle, and the label map on the right. We used Gaussian adaISA with $n=5$.

Figure 9 shows also an image from Wikimedia, the eruption 

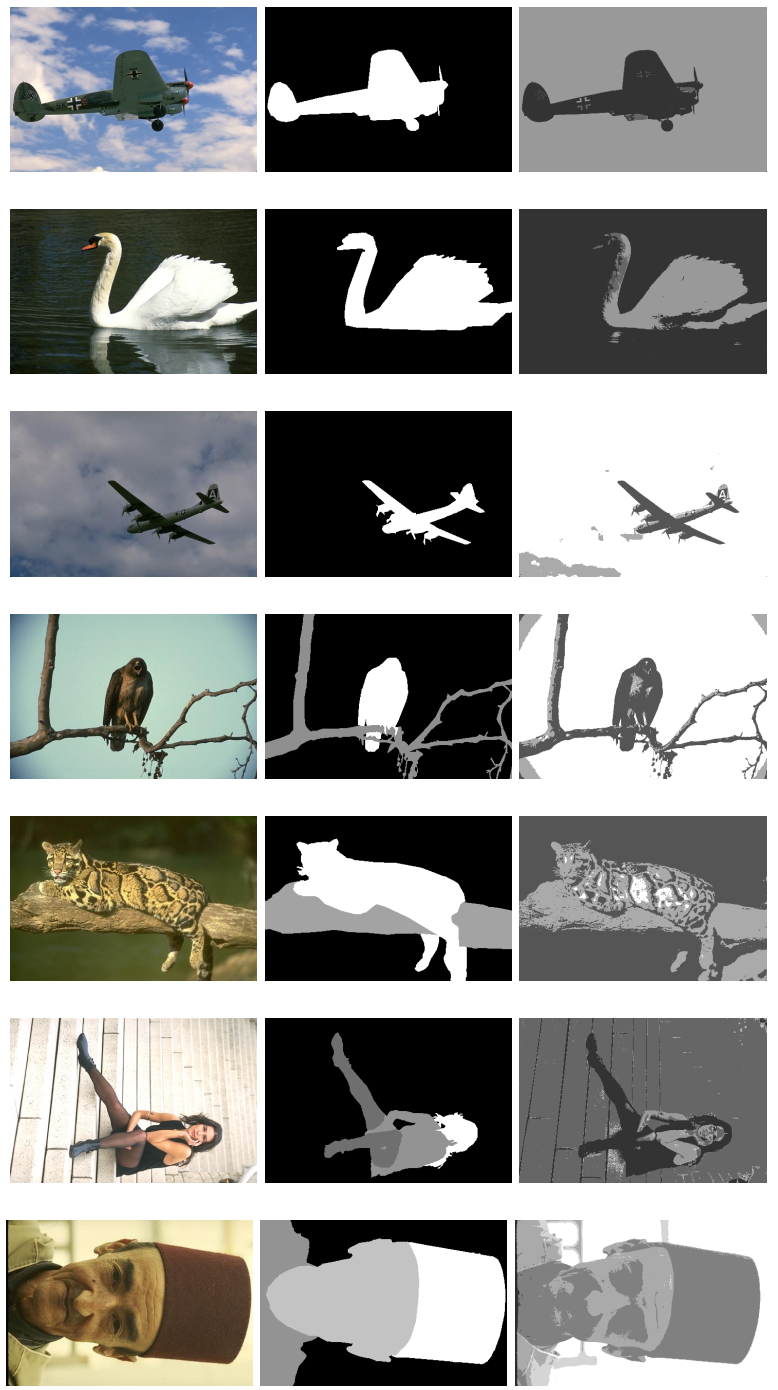

Figure 7. Gallery Left column: Original images, Center column: Ground truth images Right column: Gaussian adaISA.
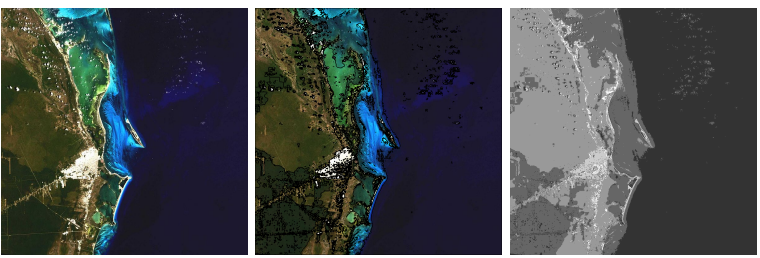

Figure 8. Left: Original Landsat_ Cancun_ Mexico from Wikimedia commons database. Middle: Segment-border image. Right: Label map

of the Calbuco volcano. Gaussian adaISA with $n=12$ can easily segment the cloud of dust and ashes of the volcano and thus compute the area affected by the eruption.

Figure 10 shows an image from Wikimedia of a dust storm
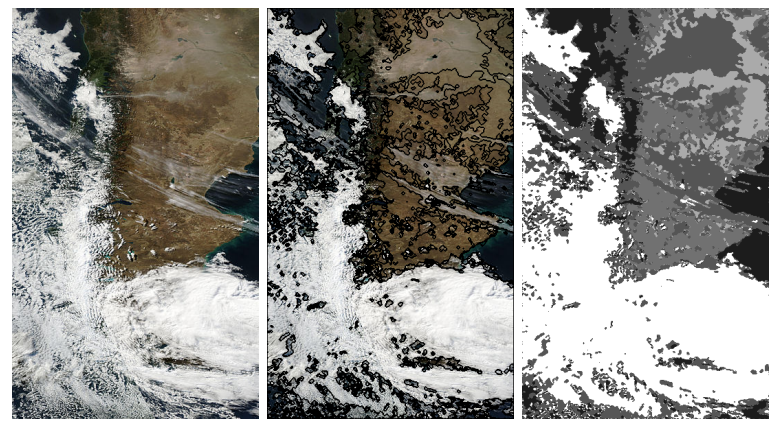

Figure 9. Left: Original Eruption_of_Calbuco_volcano from Wikimedia commons database. Middle: Segment-border image. Right: Label map
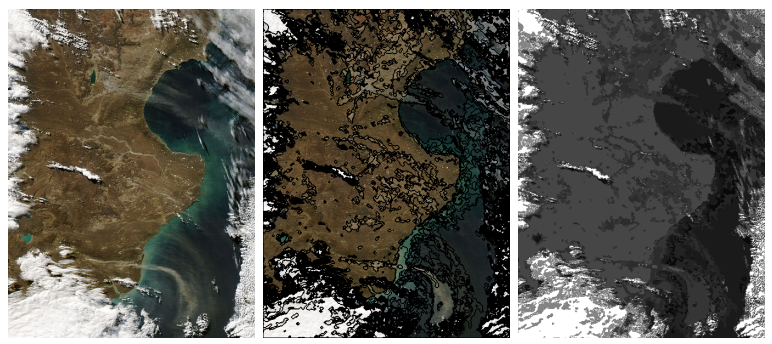

Figure 10. Left: Original Dust_storms_off_southern_Argentina from Wikimedia commons database. Middle: Segmentborder image. Right: Label map

in southern Argentina. Gaussian adaISA with $n=3$ was used to segment the image to measure the area covered by the dust storm. This input example is harder than the image of the Calbuco volcano since it is even hard for the human eye to identify the dust. The program was even identifying the dust in the lower part of the image.

The three examples illustrate the sensitivity of the proposed segmentation method if regions of only very vague value differences need to be split into different segment categories.

\section{Conclusions}

We proposed with adaISA a novel adaptive image segmentation algorithm. It is a fairly straightforward extension of common histogram-based image segmentation strategies. The proposed algorithm segments images without any prior information about the image or the number of intended segments. The user can finally decide about the number of used thresholds, for every image of processed video data, or for whole classes of images at once.

Our tests show that Gaussian ada ISA can be a good competitor for GMM and HMM-EM segmentation, with the advantage that adaISA requires less operations, is easier to program, and 
is deterministic while GMM and HMM-EM are not.

What makes adaISA special is that it follows simple concepts and applies a minimised number of steps; thus it significantly decreased computational time complexity compared to other segmentation methods while leading to comparable results. In other words, the main contribution of this paper is that the proposed algorithms lead to results similar to those obtained by HMM or GMM, but with reduced time.

The algorithm applies to any multi-channel case. For example, it can also be used for $6 D$ vision [15] where depth and optic flow contribute to the definition of the used feature space.

\section{References}

[1] P. Krähenbühl and V. Koltun, Geodesic object proposals, in ECCV, pp. 725-739, 2014.

[2] R. Klette, Concise Computer Vision: An Introduction into Theory and Algorithms, Springer London, 2014. [Online]. Available: books.google.com.mx/books?id= ZCu8BAAAQBAJ

[3] A. Qin and D. A. Clausi, Multivariate image segmentation using semantic region growing with adaptive edge penalty, IEEE Trans. Image Processing, 19:2157-2170, 2010.

[4] S. Lankton and A. Tannenbaum, Localizing region-based active contours, IEEE Trans. Image Processing, 17:20292039, 2008.

[5] P. A. Arbeláez and L. D. Cohen, A metric approach to vector-valued image segmentation, Int. J. Computer Vision, 69:119-126, 2006.

[6] N. Otsu, A threshold selection method from gray-level histograms, IEEE Trans. Systems Man Cybernetics, 9:6266, 1979.

[7] W. Feng, J. Jia, and Z.-Q. Liu, Self-validated labeling of Markov random fields for image segmentation, IEEE Trans. Pattern Analysis Machine Intelligence, 32:18711887, 2010.

[8] J. Long, E. Shelhamer, and T. Darrell, Fully convolutional networks for semantic segmentation, CVPR, pp. 34313440, 2015.

[9] J. S. Weszka and A. Rosenfeld, Threshold evaluation techniques, IEEE Trans. System Man Cybernetics, 8:622-629, 1978.
[10] R. Achanta, A. Shaji, K. Smith, A. Lucchi, P. Fua, and S. Süsstrunk, SLIC superpixels compared to state-of-theart superpixel methods, IEEE Trans. Pattern Analysis Machine Intelligence, 34:2274-2282, 2012.

[11] S. Valentine The Hidden Power of Blend Modes in Adobe Photoshop Adobe Press, 2012.

[12] L. Gupta and T. Sortrakul, A Gaussian-mixture-based image segmentation algorithm, Pattern Recognition, 31:315325, 1998.

[13] Q. Wang, HMRF-EM-image: implementation of the hidden Markov random field model and its expectationmaximization algorithm, arXiv preprint arXiv:1207.3510, 2012.

[14] W. Rand, Objective criteria for the evaluation of clustering methods, J. American Statistical Association, 66 (336): 846-850, 1971.

[15] 6D Vision, www.6d-vision.com/scene-labeling, 2016.

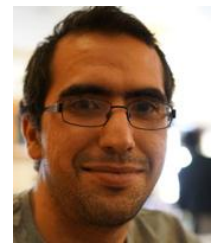

Rafael Guillermo González Acuña is a member of the Mexican Society of Physics and a member of the Mexican Academy of Optics. He has a bachelor degree in physics, a masters degree in optics, and he is currently studying for a bachelor in mathematics. He is admitted for a Ph.D. program in computer science at Pontificia Universidad Católica de Chile.

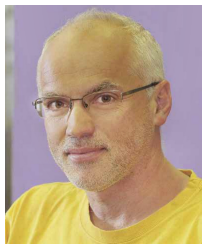

Domingo Mery is professor with the Department of Computer Science at the Pontificia Universidad Católica de Chile. He has authored over 65 technical SCI publications and over 75 conference papers. His research interests include image processing for X-ray imaging, pattern recognition, computer vision, and biometrics. He is currently Associate Editor of IEEE Transactions of Information, Forensics and Security. 


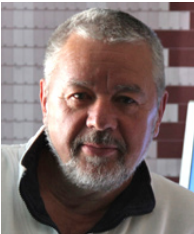

Reinhard Klette is a Fellow of the Royal So-

journals or conferences, and books on comciety of New Zealand and a professor at Auck- puter vision, image processing, geometric algorithms, and land University of Technology. He (co-)authored panoramic imaging. He was an associate editor of IEEE PAMI more than 400 publications in peer-reviewed (2001-2008). 